\title{
LDCT Screening in Smokers in India-A Pilot, Proof-of-Concept Study
}

\author{
Sanghavi Parang ${ }^{1}$ Jankharia Bhavin ${ }^{1}$ \\ 1Picture This By Jankharia, Mumbai, Maharashtra, India
}

Indian ] Radiol Imaging 2021;31:318-322.

\begin{abstract}
Address for correspondence Sanghavi Parang S, DNB, DMRD, MBBS, B-4/402, Building No 7, Ravikiran, Tilak Nagar (West)., Mumbai, Maharashtra 400089, India (e-mail: sanghaviparang@ gmail.com).
\end{abstract}

\begin{abstract}
Aim The aim of the study is to assess the effectiveness of low dose CT scan (LDCT) to pick up nodules and carcinomas in smokers in India.

Methods A retrospective study of 350 smokers scanned with LDCT was performed in a private practice center in Mumbai, India. Their demographic profile, smoking history in pack-years, lung nodules, and associated findings/superimposed complications were recorded and analyzed. The nodules were assigned the appropriate Lung-RADS category and results of the patients undergoing biopsy were recorded.

Results Of the 350 smokers, 15 (4\%) were women. The mean age was 61 years (32-90 years) with a mean smoking history of 22 pack-years (1-160 pack-years). Lung nodules were found in 335 (93\%) smokers, with Lung-RADS category 1 nodules seen in 117 (36\%), category 2 in $133(41 \%)$, category 3 in 29 (9\%), and category 4 in $46(14 \%)$ of positive scans. Seven of the category 4 patients who underwent biopsy showed carcinoma, with a mean smoking history of 30 pack years. Superimposed interstitial lung disease, airways diseases, and infections were also seen in the scans and recorded.

Discussion It has been proven in the Western world that screening for lung cancer with LDCT saves lives with early pick-up of malignant nodules. This pilot study from

Keywords

- LDCT

- screening

- smokers

- nodules

- lung cancer India, shows that even in a tuberculosis endemic country, LDCT picks up malignant lung nodules early and saves lives.

Conclusion Smokers with a more than 20 pack years history have a higher incidence of lung nodules, which may represent carcinoma, in a tuberculosis endemic country like India, as has been proven in other countries in the Western world. Larger studies and trials may be performed to elaborate further on this proof-of-concept study.
\end{abstract}

\section{Aims and Objectives}

\section{Primary Aim}

- To assess the effectiveness of low dose CT scan (LDCT) to pick up nodules and carcinomas in smokers.

\section{Secondary Aims}

- To understand the demographics of smokers and lung cancers in India.

published online

July 28, 2021
DOI https://doi.org/

10.1055/s-0041-1734227

ISSN 0971-3026
- To evaluate the distribution of various lung nodules according to the Lung-RADS criteria categories.

- To calculate the risk of cancer in the various categories lung nodules.

\section{Introduction}

Lung cancer is an aggressive and heterogenous disease. It constitutes approximately $6.9 \%$ of all new cancer cases

(c) 2021. Indian Radiological Association.

This is an open access article published by Thieme under the terms of the Creative Commons Attribution-NonDerivative-NonCommercial-License, permitting copying and reproduction so long as the original work is given appropriate credit. Contents may not be used for commercial purposes, or adapted, remixed, transformed or built upon. (https://creativecommons.org/licenses/by-nc-nd/4.0/).

Thieme Medical and Scientific Publishers Private Ltd. A-12, Second Floor, Sector -2, NOIDA -201301, India 
and $9.3 \%$ of all cancer-related deaths in India. ${ }^{1}$ Globally, lung cancer comprises approximately $13 \%$ of all new cancers cases and $19 \%$ of all cancer-related deaths. ${ }^{2}$

$70 \%$ of the patients with lung cancer present with locally advanced or metastatic disease. While many advances in surgical, chemotherapeutic, and radiotherapeutic treatments have been made in past few years, the overall 5-year survival of nonsmall cell lung cancer is only $15 \%$. If detected early, the 5-year survival is $57 \%{ }^{3}$ Hence it is very important to diagnose lung cancer early to improve survival.

Lung cancer is associated with a variety of risk factors like smoking, family history of lung cancer, various environmental and occupational exposures like radon, chronic obstructive pulmonary disease (COPD) and interstitial lung disease (ILD), and thoracic radiation, among others. Smoking is the single most common cause of lung cancer. ${ }^{4}$

Screening with CT scan has been proven to reduce mortality from lung cancer in high-risk populations. The National Lung Screening Trial (NLST) 5 showed a $20 \%$ reduction in mortality when low dose CT scan (LDCT) was used for screening. LDCT has also been shown to be effective in tuberculosis endemic countries as well as in real-life situations such as ours. ${ }^{6,7}$

\section{Materials and Methods}

A retrospective study of 350 smokers scanned with LDCT over a period of 3 years from January 2016 to January 2019 was performed at a private practice center in Mumbai, India. The study was approved by Institutional Review Board and the written informed consent was waived off.

\section{Inclusion Criteria}

\section{Smoker}

\section{Exclusion Criteria}

1. Known lung malignancy.

2. Known malignancy elsewhere in body.

3. Stopped smoking $>15$ years.

The demographic profile of every subject, including age and gender was recorded. A detailed smoking history was recorded: current or ex-smoker and pack-years of smoking.

The primary aim was to record the presence or absence of lung nodules and the number and exact location of each nodule found. The largest nodule was assigned a category as per the Lung-RADS criteria. ${ }^{8}$ A specific management plan was assigned including follow-up interval, PET/CT, or tissue sampling with biopsy. The histopathological findings of all subjects undergoing biopsy were recorded.

Any associated findings related to smoking including superimposed complications were also recorded.

\section{Low Dose CT (LDCT) Chest Protocol}

All the scans were performed on a Siemens Somatom Definition Edge CT scanner with $120 \mathrm{kV}$ and $50 \mathrm{mAs}$ or less (effective $\mathrm{mAs}$ ) optimized to deliver an effective dose of less than $1.5 \mathrm{mSv}$ with pitch of 1.2. The scan was acquired from the thoracic inlet to the costophrenic angles with breath-hold in full suspended inspiration, obtained after adequate prior training. The data was reconstructed both in lung and soft tissue reconstruction algorithms with axial volumetric data with $1-\mathrm{mm}$ slice thickness at $0.5-\mathrm{mm}$ intervals.

The radiation dose through an LDCT scan was $1.5 \mathrm{mSv}$, as per international recommendations. ${ }^{9}$

\section{Results}

A total of 350 smokers who underwent LDCT from January 2017 to January 2020 were studied.

The age range was from 32 to 90 years. Their mean age was 61 years. There were 335 men and 15 women. The men had an age range of 32 to 90 years with a mean age of 61 years. The women's age range was 37 to 53 years with a mean age of 45 years ( - Table 1). As evident from - Fig. 1, the majority of the subjects in this study were between 61 to 70 years of age. As evident from - Fig. 2, the majority of the subjects in this study were men (95.7\%).

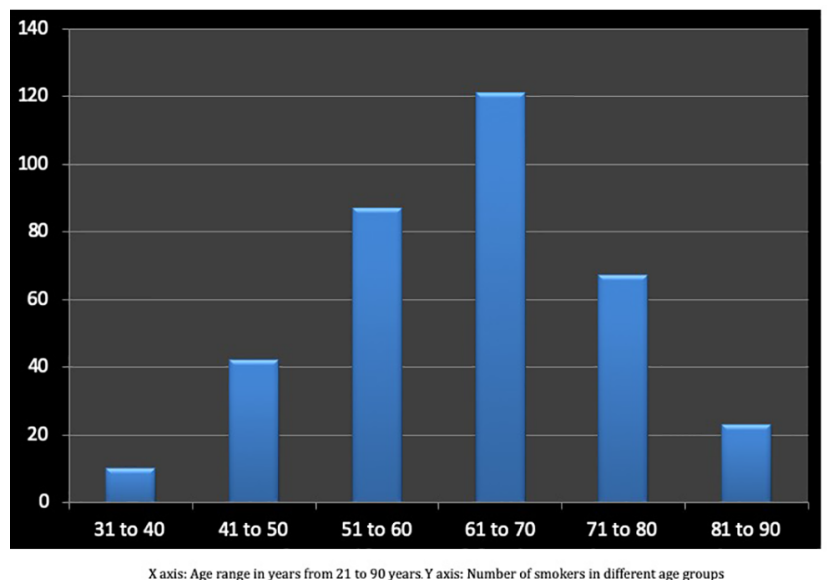

Fig. 1 Age distribution of subjects. $X$ axis: Age range in years. $Y$ axis: Number of smokers in different age groups. The age range in our study was 32 years to 90 years with majority of the subjects in 61 to 70 years age group.

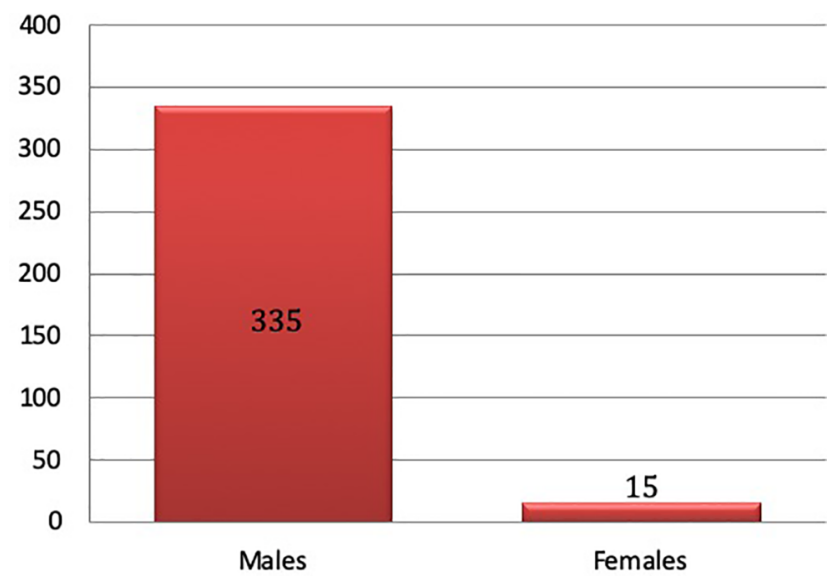

Fig. 2 Gender distribution of subjects. The majority of the subjects $(95.7 \%)$ were males in our study. 
The smoking history ranged from 1 to 160 pack-years with a median value of 22 pack-years. The category 4 subjects had a mean smoking history of 30 pack-years.

At least one lung nodule was found in 325 subjects, i.e., in $93 \%$ of the studies ( - Fig. 3 ). Only 25 subjects, i.e., $7 \%$ of the studies did not show a lung nodule.

In the rest of the 325 positive scans, a Lung-RADS category was assigned to the largest nodule ( - Fig. 4 ). We found a category 1 nodule in 117 scans (36\%), category 2 in 133 scans (41\%), category 3 in 29 scans (9\%) and category 4 in 46 scans (14\%) (-Figs. 5 and 6). Category 2 nodules were the commonest and category 3 nodules were the least commonly seen of all the categories.

Seven subjects with category 4 nodules who underwent biopsy showed adenocarcinoma ( - Fig. 7 ), with a $2 \%$ incidence of lung cancer ( - Fig. 8).

\section{Discussion}

Lung cancer constitutes approximately $6.9 \%$ of all new cancer cases and $9.3 \%$ of all cancer-related deaths in India. ${ }^{1}$ Worldwide, there were estimated approximately 1.6 million deaths from lung cancer in $2012,70 \%$ of these in men. ${ }^{2}$ Seventy-five percent of patients with lung cancer present with locally advanced or metastatic disease and the overall 5-year survival of nonsmall cell lung cancer is just $15 \%$. If detected early,

\section{$\square$ Found $\quad$ Not Found}

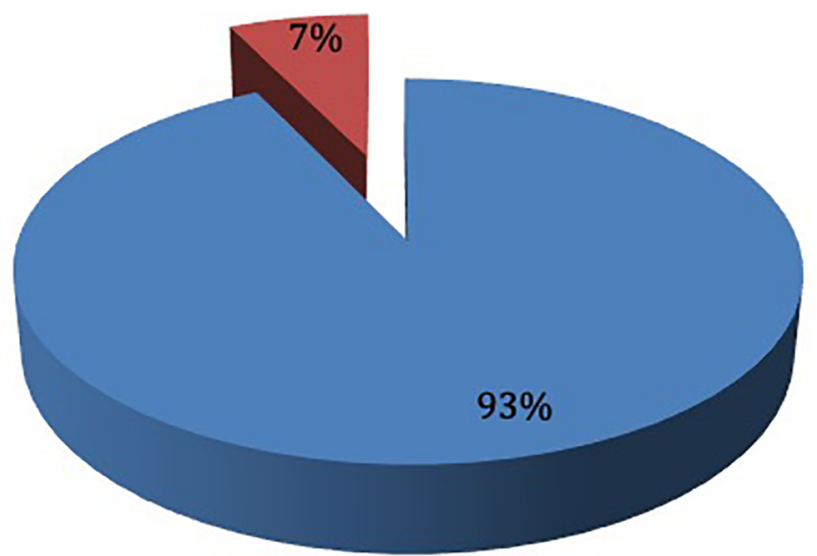

Fig. 3 Lung nodules incidence. The lung nodule incidence in the study was very high, $93 \%$ and a lung nodule was found in 325 subjects.
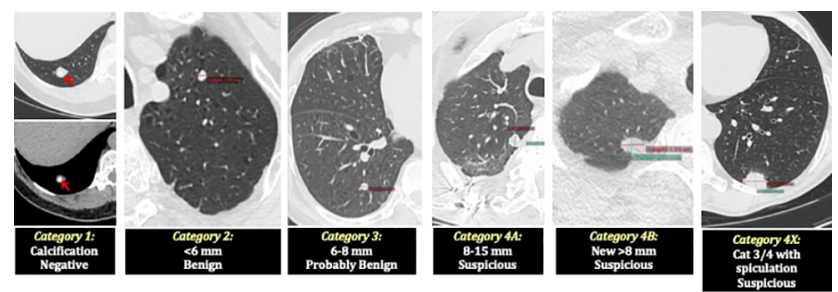

Fig. 4 Lung Nodules found in our study. The various Lung RADS categories nodules found in our study from categories 1 to 4 are shown including subdivisions of $4 \mathrm{~A}, 4 \mathrm{~B}$ and $4 \mathrm{X}$.

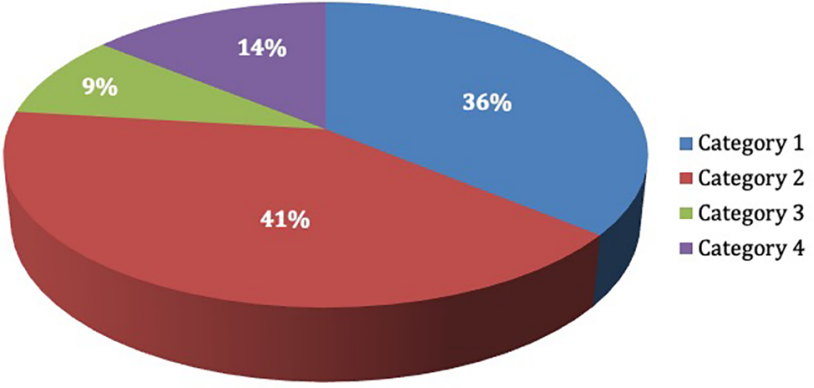

Fig. 6 Lung nodules distribution. In our study, Category 2 nodules were most common and found in $41 \%$ of subjects, followed by Category 1 nodules which were found in $36 \%$ of subjects. Category 4 nodules were found in 14\% of subjects and Category 3 nodules were lease common which were found in only $9 \%$ of subjects.

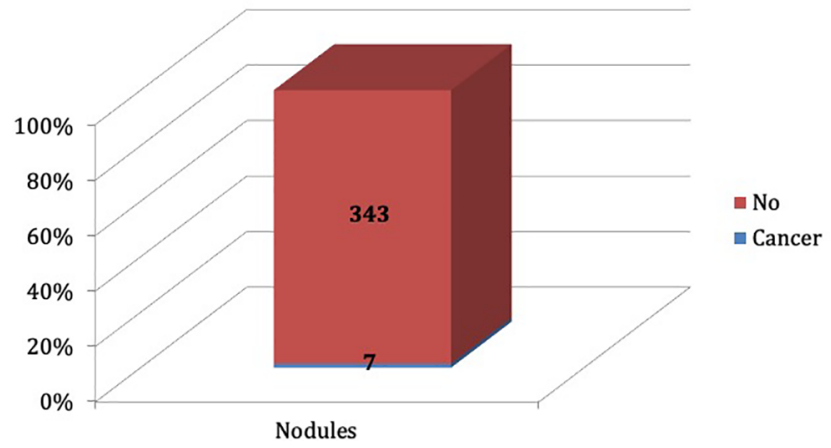

Fig. 7 Frequency of Cancers. In our study, we found cancer in total 7 subjects, and all were category 4 nodules.

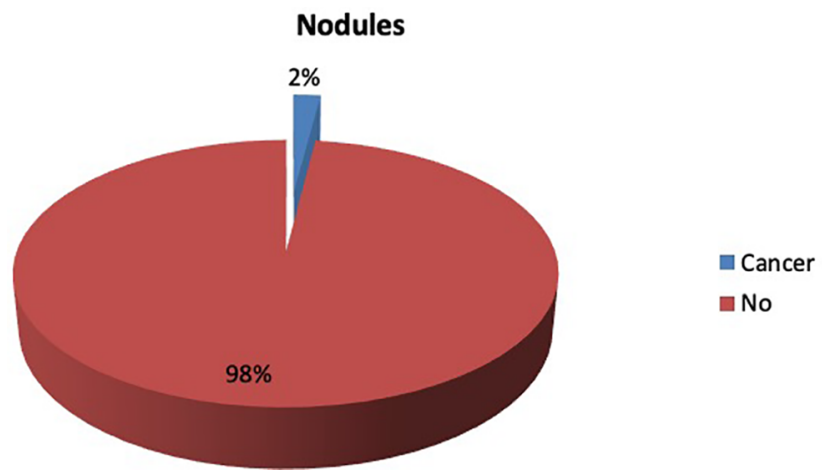

Fig. 8 Incidence of Cancers. In our study, we found 2\% incidence of lung cancer.

the 5 -year survival is $57 \%{ }^{3}$ Hence early diagnosis can lead to early curative treatment and reduction in mortality.

Though lung cancer is caused by variety of risk factors, smoking remains the single most common cause of lung cancer. ${ }^{4}$ Hence, smokers are the target population for screening for lung cancer. India ranks third in global tobacco production and consumes almost $50 \%$ of its products domestically..$^{10}$ Smoking is directly responsible for almost $90 \%$ of lung cancer and COPD deaths. ${ }^{11}$ Screening with CT scan is the only test that has been proven to reduce mortality from lung cancer in high-risk populations. ${ }^{5}$ 
Table 1 Total of 350 smokers were studied. The age range was from 32 to 90 years with mean age of 61 years. For 335 male subjects, the age range was 32 to 90 years and the mean age was 61 years. For 15 female subjects, the age range was 37 to 53 years and the mean age was 45 years.

\begin{tabular}{|l|l|l|l|l|}
\hline & Number & $\begin{array}{l}\text { Minimum age } \\
\text { (years) }\end{array}$ & $\begin{array}{l}\text { Maximum age } \\
\text { (years) }\end{array}$ & Mean age (years) \\
\hline Male & 335 & 32 & 90 & 61 \\
\hline Female & 15 & 37 & 53 & 45 \\
\hline Total & 350 & 32 & 90 & 61 \\
\hline
\end{tabular}

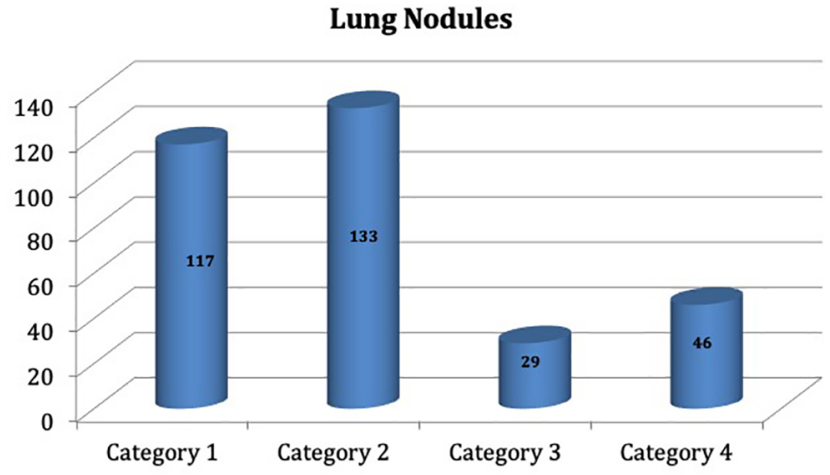

Fig. 5 Lung nodules distribution. In our study, Category 2 nodules were most common and found in 144 scans, followed by Category 1 nodules which were found in 126 scans. Category 4 nodules were found in 49 scans and Category 3 nodules were least common which were found in only 31 scans.

The NLST ${ }^{5}$ showed a lung cancer prevalence of $1 \%$ in the screened population with the vast majority (63\%) of the lung cancers in stage I and a $20 \%$ reduction in mortality in this population. The International Early Lung Cancer Action Program (I-ELCAP) ${ }^{12}$ showed that the curability of Stage I lung cancers is 80 to $90 \%$ and annual CT screening allows at least $80 \%$ of lung cancers to be diagnosed at clinical Stage I. breast, cervical, and colon cancer screenings. The Nelson trial, ${ }^{13}$ the second largest randomized control trial (RCT) after the NLST, showed that $50 \%$ of the cancers diagnosed in the screening arm were early stage and 65 to $70 \%$ were stages IA to II, while approximately $70 \%$ of cancers in the control arm were stage III/IV at diagnosis. Overall, CT scanning decreased mortality by $26 \%$ in high-risk men and up to $39 \%$ in high-risk women over a period of 10 years.

The recent Korean Lung Cancer Screening Project (K-LUCAS) ${ }^{6}$ showed that LDCT screening in tuberculosis-endemic country may be less effective because of false-positive results and hence lower specificity caused by tuberculosis sequelae. Tuberculosis sequelae were associated with positive CT screening results, but no evidence was found of an association with lung cancer. Another recent study to assess real world LDCT lung cancer screening performance showed that adherence to recommendations was increased in former than current smoker and in those with positive lung cancer screening exam. ${ }^{7}$

Our study group included subjects with ages ranging from 32 to 90 years with a mean age of 61 years. As expected, $95.7 \%$ were men. With LDCT, we found at least one lung nodule in 325 subjects (93\%). Category 2 nodules were the commonest and were seen in 144 scans (41\%), with category 4 nodules seen in 46 scans (14\%).

As per management recommendations, seven of the subjects with category 4 nodules underwent CT-guided core biopsy and we found lung cancer all seven of them, six adenocarcinoma, and one small cell lung cancer, with an overall lung cancer incidence of $2 \%$.

This pilot, proof-of-concept study showed preliminary results consistent with the previously mentioned trials. Our study has many limitations. It was retrospective, nonrandomized, with a small sample size and confined to a single center. However, the aim was to show that LDCT works even in a tuberculosis endemic country like India, and we hope that this pilot study spurs the development of larger RCTs. A recent paper by Shankar et al also highlights the importance and challenges of a successful LDCT program in a tuberculosis endemic country like India. ${ }^{14}$

There are many benefits of LDCT for lung cancer screening trial. As discussed, LDCT saves lives by early detection of the lung cancer, thus reducing mortality. LDCT can pick up other smoking-related lung diseases like COPD, ILD, coronary artery disease, etc., which can then be adequately treated to prevent morbidity. It can also be a starting point for encouraging smoking cessation. However, there are potential risks too like cancer may not be always detected early enough to be treated or sometimes indolent cancers may get treated which might have not caused death. It leads to anxiety in the subjects. Radiation is also a potential risk but with low dose protocol the risk is further reduced. False positive and false negative results may occur which may lead to harmful effects. Financial costs also need to be addressed as the target population is large.

\section{Conclusion}

Our pilot, proof-of-concept study shows that LDCT in a tuberculosis endemic country like India can also pick up lung cancer early and save lives.

\section{Presentation at a Meeting}

None.

\section{Funding Sources \\ None.}

\section{Conflict of Interest}

None declared. 


\section{Acknowledgments}

None.

\section{References}

1 https://ncdirindia.org/All_Reports/PBCR_REPORT_2009_ 2011/ALL_CONTENT/PDF_Printed_Version/Preliminary_ Pages_Printed.pdf

2 Ferlay J, Soerjomataram I, Dikshit R, Eser S, Mathers C, Rebelo M, Parkin DM, Forman D, Bray F. Cancer incidence and mortality worldwide: sources, methods and major patterns in GLOBOCAN 2012. Int J Cancer. 2015 Mar 1;136(5):E359-86

3 American Cancer Society. Cancer facts \& figures 2020. Available at: https://www.cancer.org/research/cancer-factsstatistics/all-cancer-facts-figures/cancer-facts-figures-2020. html Accessed March 16, 2020

4 Youlden DR, Cramb SM, Baade PD. The International Epidemiology of Lung Cancer: geographical distribution and secular trends. J Thorac Oncol 2008;3(8):819-831

5 Aberle DR, Adams AM, Berg CD, et al; National Lung Screening Trial Research Team. Reduced lung-cancer mortality with low-dose computed tomographic screening. N Engl J Med 2011;365(5):395-409

6 Kim H, Kim HY, Goo JM, Kim Y. Lung cancer CT screening and lung-RADS in a tuberculosis-endemic country: The Korean Lung Cancer Screening Project (K-LUCAS. Radiology 2020;296(1):181-188

7 Barbosa EJM Jr, Yang R, Hershman M. Real-World Lung Cancer CT screening performance, smoking behavior, and adherence to recommendations: Lung-RADS category and smoking status predict adherence. AJR Am J Roentgenol 2021;216(4):919-926

8 American College of Radiology. Lung CT Screening Reporting \& Data System (Lung-RADS). Available at: https://www.acr.org/ Clinical-Resources/Reporting-and-Data-Systems/Lung-Rads Accessed February 12, 2020

9 Mascalchi M, Sali L. Lung cancer screening with low dose CT and radiation harm-from prediction models to cancer incidence data. Ann Transl Med 2017;5(17):360

10 Statista. Leading tobacco producing countries worldwide in 2019 (in 1,000 metric tons). ${ }^{*}$ Available at: https://www. statista.com/statistics/261173/leading-countries-in-tobacco-production Accessed March 17, 2020

11 Jamal A, Phillips E, Gentzke AS, et al; Centers for Disease Control and Prevention. Current cigarette smoking among adults-United States, 2016. MMWR Morb Mortal Wkly Rep 2018;67(2):53-59

12 Henschke CI. Early Lung Cancer Action Project: overall design and findings from baseline screening. The Lancet. 1999;354(9173):99:105

13 De Koning H, Van Der Aalst C, Ten Haaf K, et al. Effects of volume CT lung cancer screening: mortality results of the NELSON randomized-controlled population-based trial. Paper presented at: 2018 World Conference on Lung Cancer. Abstract PL02.05; 2018

14 Shankar A, Saini D, Dubey A, et al. Feasibility of lung cancer screening in developing countries: challenges, opportunities and way forward. Transl Lung Cancer Res 2019;8(suppl 1): S106-S121 